\title{
Methodische Untersuchungen zur Bestimmung der Plasmacorticoide mit der Proteinbindungsmethode
}

\author{
Von J. Köbberling und A. v. z. Mühlen \\ Mediqinische Universitätsklinik Göttingen, Arbeitsgruppe Endokrinologie
}

(Eingegangen am 30. August 1971)

\begin{abstract}
Es wird über eine Methode der Cortisolbestimmung nach dem Prinzip der kompetitiven Proteinbindung berichtet, die sich von bisher beschriebenen Verfahren hauptsächlich durch eine günstigere Zusammensetzung des ${ }^{3} \mathrm{H}$-Cortisol-Bindungsplasmas und durch die Anwendung einer Repetierspritze zum schnellen Einpipettieren der Dextran-Holzkohleaufschwemmung unterscheidet. Durch die Untersuchung möglicher Fehlerquellen konnte die Störanfälligkeit verringert werden. Dic Methode zeichnet sich durch ausreichende Spezifität, hohe Präzision, sehr hohe Empfindlichkeit und vergleichsweise geringen Arbeitsaufwand aus. Sie cignet sich auch zur Bestimmung des Cortisols auf Kapillar-Vollblut, der freien Corticoide aus dem Urin, des Corticosterons im Rattenplasma und mit leichten Veränderungen des Plasma-11-Desoxycortisols und des Progesterons. Anhand von Normalwerten unter verschiedenen Bedingungen konnte gezeigt werden, daß die Methode sich gut für Untersuchungen der Nebennierenrindenfunktion eignet. Dic Trennung zwischen Normalwerten und erniedrigten Werten (z. B. nach Dexamethason) ist schärfer als mit der fluorimetrischen Methode. Die Normalwerte liegen um durchschnittlich $23 \%$ niedriger. Im übrigen findet sich eine gute Ubereinstimmung zwischen den beiden Methoden.
\end{abstract}

\section{Study of a protein binding metbod for the determination of plasma corticoids}

A method, based on the principle of competitive protein binding, is described for the determination of cortisol. Compared with hitherto described methods, it employs a more favorable composition for the ${ }^{3} \mathrm{H}$-cortisol-binding plasma and an automatic pipette is used for the rapid introduction of the dextran-charcoal mixture. Possible sources of error were investigated and the likelihood of interference was minimised. The method has a satisfactory specificity, high precision, very high sensitivity and requires comparatively little time. It is also suitable for the determination of cortisol in capillary whole blood, free corticoids in urine, corticosterone in rat plasma and, with slight modification, for the measurement of plasma 11-deoxycortisol and progesterone. Using normal values under different conditions, it was shown that the method is well suited to the study of adrenal cortex function. The differentiation between normal values and lowered values (e. g. after dexamethason) is sharper than with the fluorometric method; the average normal values are $23 \%$ lower. Furthermore, there is good agreement between the two methods.

Seit der ersten Mitteilung von Ekrss (1) über die Möglichkeit, mit Hilfe von Verdrängungsanalysen an Bindungsproteinen einen sehr empfindlichen Nachweis verschiedener Substanzen zu führen, sind Proteinbindungsmethoden für Cortisol, 11-Desoxycortisol, Corticosteron, Progesteron, 17-Hydroxyprogesteron, Testosteron, Östrogene, Aldosteron und andere Steroidhormone beschrieben worden (2). Das Prinzip der Methode beruht darauf, daß konstante Mengen radioaktiv markierter und variable Mengen nicht markierter Substanz um die Ligandenstellen des Bindungsproteins kompetieren und $\mathrm{da} \beta$ nach Trennung von gebundenem und ungebundenem Anteil aus eingegebenen bekannten Mengen eine Standardkurve gezeichnet werden kann, mit deren Hilfe in gleicher Weise behandelte unbekannte Mengen abgelesen werden können.

Wir glauben, daß das System der kompetitiven Proteinbindung für Plasma-Cortisol als klinische Routinemethode geeignet ist. Verschiedene klinisch brauchbare Methoden sind beschrieben worden, die sich jeweils nur in Details unterschieden (3-7). Wir haben einige Verbesserungen und technische Vereinfachungen eingeführt, die die Anwendbarkeit für die Routinediagnostik erleichtern. Daneben galten unsere Untersuchungen besonders der Frage nach möglichen Fehlerquellen und dem Vergleich mit der in der Routine bislang üblicherweise verwandten fluorometrischen Methode (8). Dar- über hinaus wollen wir auf einige weitere Möglichkeiten, die diese Proteinbindungsmethode bietet, hinweisen.

\section{Material und Methoden}

\section{Reagenzien}

Dichlormethan „Uvasol“ (Merck AG, Darmstadt)

Äthanol absolut (Merck AG, Darmstadt)

Aktivkohle reinst (Merck AG, Darmstadt)

Dextran 70 (Pharmacia, Frankfurt)

Insta-Gel (Packard Instrument Comp., Frankfurt)

Hydrocortison (Schering AG, Berlin)

Hydrocortison-[1, $\left.2^{3} \mathrm{H}\right], 20-30 \mathrm{C} / \mathrm{mMol}$ (NEN-Chemicals, Dreieichenhain)

Phosphatpuffer 0,15 $\mathrm{M} \mathrm{pH} \mathrm{7,4}$

Dextran-Holzkohle-Aufschwemmung:

$\begin{array}{ll}\text { Dextran . } & 0,05 \\ \text { Aktivkohle } & 0,5 \\ \text { Phosphatpuffer ad } & 150\end{array}$

(bei $+4^{\circ}$ mehrere Wochen haltbar)

Cortisol-Standard:

Hydrocortison $1 \mathrm{mg} / \mathrm{l}$ in absolutcm Äthanol, in kleinen Portionen vor Gebrauch auf $100 \mu \mathrm{g} / 1$ verdünnen.

\section{Herstellung des ${ }^{3} \mathrm{H}$-Cortisol-Bindungsplasmas}

Von einer Blutspenderin, die Ovulationshemmer cinnimmt, wird nach Gabe von $3 \mathrm{mg}$ Dexamethason am Vorabend $500 \mathrm{ml}$ Blut entnommen und das Plasma abzentrifugicrt. Jetzt wird eine zunächst geringe Menge von ${ }^{3} \mathrm{H}-$ Cortisol zugeführt und mit dem Plasma in unten angegebener Weise cine Standardkurve mit 
vielen Einzelpunkten bestimmt. $\mathrm{Da}$ das Transcortin noch nicht mit Cortisol abgesättigt ist, ergibt sich zunächst eine s-förmige Kurve, also eine zusätzliche Abflachung der Kurve im niedrigen Bercich (Abb. 1). An dieser s-förmigen Kurve wird die Cortisolmenge am Wendepunkt der Kurve, also an dem Punkt, an dem der steilste Abfall beginnt, abgelesen. Diese Cortisolmenge wird nun dem Plasma als ${ }^{3} \mathrm{H}$-Cortisol, bei sehr großen Mengen auch teilweise als nicht markiertes Cortisol zugesetzt (da die Kurve in Abbildung 1 bei einer Plasmaverdünnung von 1:150 ge-

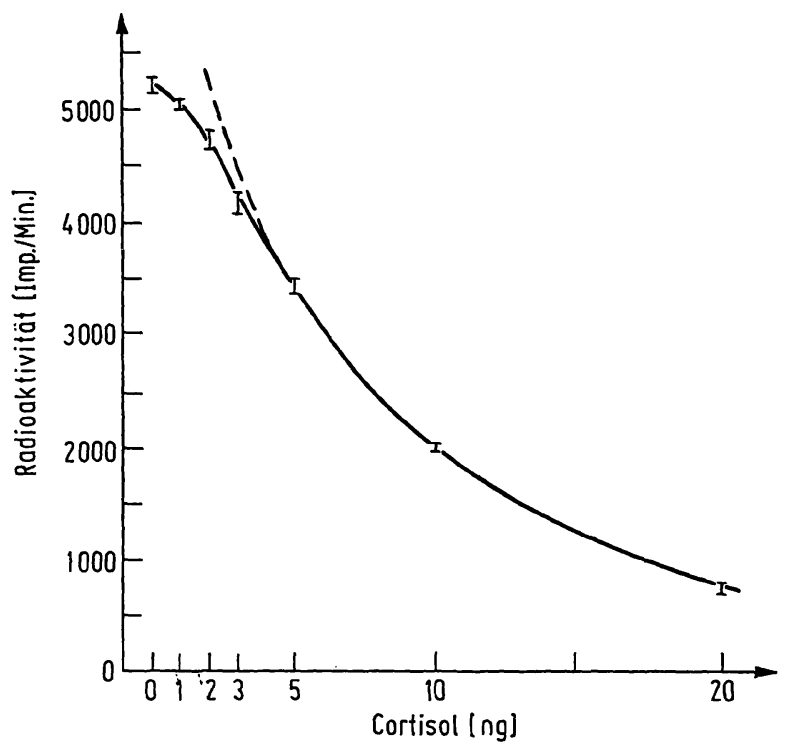

Abb. 1

Cortisol-Standardkurve bei nicht abgesättigtem Transcortin (6-fachBestimmungen)

Der Wendepunkt der Kurve bei etwa $4 \mathrm{ng}$

wonnen wurde, in diesem Falle also $600 \mathrm{ng}$ Cortisol pro $\mathrm{ml}$ Bindungsplasma). Wegen der Notwendigkeit dieser Vortestung setzen wir jeweils eine größere Menge des ${ }^{3} \mathrm{H}$-Cortisol-Bindungsplasmas in Verdünnungen von 1:3 mit dest. Wasser an, das wir in Portionen zu jewreils $4 \mathrm{ml}$ (also $1 \mathrm{ml}$ Plasma entsprechend) in Plastikgefäße abfüllen und gefriertrocknen. Im Laufe von 8 Monaten haben wir keine Veränderungen in den Eigenschaften dieses lyophilisierten Plasmas beobachtet. Auch tiefgefroren hält es sich zumindest einige Wochen. Die $1 \mathrm{ml}$ Plasmaportionen werden zum Gebrauch mit der jeweils gewünschten Menge Puffer aufgelöst, bei uns in der Routine mit $150 \mathrm{ml}$. In dieser Form ist es nur einige Tage haltbar. Die Zusammensetzung unseres letzten Bindungsplasmas lautet:

Plasma $1 \mathrm{ml}$,

Hydrocortison- $\left[1,2{ }^{3} \mathrm{H}\right] 50 \mu \mathrm{C}$ (615 ng),

Hydrocortison $180 \mathrm{ng}$

Phosphatpuffer ad $150 \mathrm{ml}$.

\section{Vorgeben}

Alle Glassachen müssen gründlich gereinigt und mit Alkohol gespült sein. In $10 \mathrm{ml}$ Extraktionsgläsern mit Schliffstopfen wird $0,1 \mathrm{ml}$ Plasma (bei Proben nach ACTH $0,05 \mathrm{ml}$ ) mit $2 \mathrm{ml}$ Dichlormethan durch $30 \mathrm{Sek}$. Schütteln mit Vortex-Mischer extrahiert. $1 \mathrm{ml}$ des Dichlormethan-Extraktes wird in ein Zentrifugenglas überpipettiert. In weitere Zentrifugengläser werden mit Konstriktionspipetten Standardmengen von $0,2,5,10$ und $20 \mathrm{ng}$ des Cortisolstandards $(0,20,50,100$ und $200 \mu \mathrm{l}$ der Standardlösung) pipettiert. Alle Standards und Plasmaextrakte werden als Doppelwerte angesetzt. Die maximale Anzahl der Einzelproben wird durch die Größe der Zentrifugeneinsätze begrenzt. Die Standardwerte und die Plasmaextrakte werden im Luftstrom im Wasserbad zur Trockne gebracht. Wir verwenden hierzu einen aus Glas gefertigten Verteilerrechen für den .Luftstrom, passend für die Zentrifugengläser im Reagenzglasständer. Anschließend wird in jedes Glas mit einer Repetierspritze (,Ultra-
Asept ${ }^{\text {cc }}, 2 \mathrm{ml}$, Fa. Henke, Tuttlingen) $1 \mathrm{ml}$ der ${ }^{3} \mathrm{H}$-CortisolPlasmalösung eingegeben. Alle Gläser werden einige Sek. mit dem Vortex-Mischer und anschließend $5 \mathrm{Min}$. bei $37^{\circ} \mathrm{im}$ Wasserbad geschüttelt. Die Proben werden dann für $20 \mathrm{Min}$. im Eiswasser inkubiert. Nach dieser Zeit wird mit der Repetierspritze $0,5 \mathrm{ml}$ der Dextran-Holzkohle-Aufschwemmung pipettiert. Dieser Pipettiervorgang muß so schnell wie möglich exfolgen. Dié Kohleaufschwemmung mit einem Magnetrührkern steht während dieser Zeit ebenfalls in einem Eisbad auf einem automatischen Magnetrührer. Auch die Repetierspritze wird im Eisbad vorgekühlt. Von der ersten Probe wird mindestens $20 \mathrm{mal}$ leer pipettiert.

Anschließend werden die Proben gemeinsam im Ständer einige Sek. kräftig mit der Hand geschüttelt und noch weitere $10 \mathrm{Min}$. im Eiswasser gekühlt. Während dieser Zeit werden auch die Zentrifugeneinsätze vorgekühlt (die Vorkühlung kann bei Vorhandensein einer Kühlzentrifuge entfallen). Die Proben müssen dann sofort gleichzeitig etwa $5 \mathrm{Min}$. bei etwa $2000 \mathrm{~g}$ zentrifugiert werden. Auch nach dem Zentrifugieren werden die Zentrifugeneinsätze mit den Proben wieder ins Eisbad gestellt. Von dem Überstand werden $0,5 \mathrm{ml}$ mit einer Eppendorf-Mikroliterpipette in ein Plastik-Zählgefäß mit $10 \mathrm{ml}$ Insta-Gel als Szintillationslösung pipettiert. Gezählt wird nach $30 \mathrm{Min}$. Vorkühlung mit einer Zeitvorgabe von $1 \mathrm{Min}$. oder einer Impuls-Vorgabe von 50000 .

Nach den abgelesenen Impulsen wird aus den Standardmengen eine Standardkurve im linearen Maßstab gezeichnet. An dieser Kurve werden die unbekannten Proben abgelesen. Die abgelesenen ng-Werte werden mit 20 multipliziert (bei $0,05 \mathrm{ml}$ Plasma mit 40) und ergeben dann den Plasmagehalt an Cortisol in $\mathrm{ng} / \mathrm{ml}$ bzw. $\mu \mathrm{g} / \mathrm{l}$.

\section{Ergebnisse}

Abbildung 2 zeigt eine Standardkurve im Bereich von $0-20 \mathrm{ng}$ mit unserem ${ }^{3} \mathrm{H}$-Cortisol-Bindungsplasma in der üblichen Verdünnung von 1:150. Bei den angegebenen Impulsen/Minute zwischen 13000 und 52000 liegt der mittlere Fehler der Zählung bei einer Zählzeit von $1 \mathrm{Min}$. zwischen 0,3 und $0,7 \%$. Die Gesamtstreuung lag bei einer 12fach-Bestimmung bei etwa 1,2-1,5\%, so $\mathrm{da} ß$ der angegebene Zählfehler vertretbar ist. Die Zählung einer Serie von 72 Proben nimmt also nur etwas mehr als eine Stunde in Anspruch. Dieselbe Standardkurve läßt sich auch in der Form zeichnen,

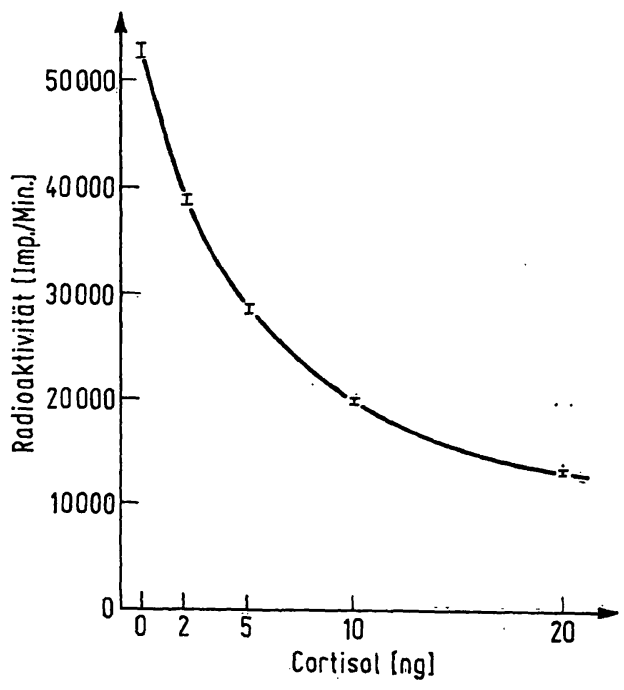

Abb. 2

Cortisol-Standardkurve (12fach-Bestimmungen) mit dem ${ }^{3} \mathrm{H}-\mathrm{Cor}$ tisol-Bindungsplasma in oben angegebener Zusammensetzung 
daß der mittlerc Nullwert $\left(C_{0}\right)$ durch alle Einzelwerte $\left(C_{1}\right)$ geteilt wird (Abb. 3). Die Werte liegen dann, wie aus thcoretischen Uberlegungen (9) zu erwarten ist, in cinem weiten Bereich auf einer Geraden. Auch bei

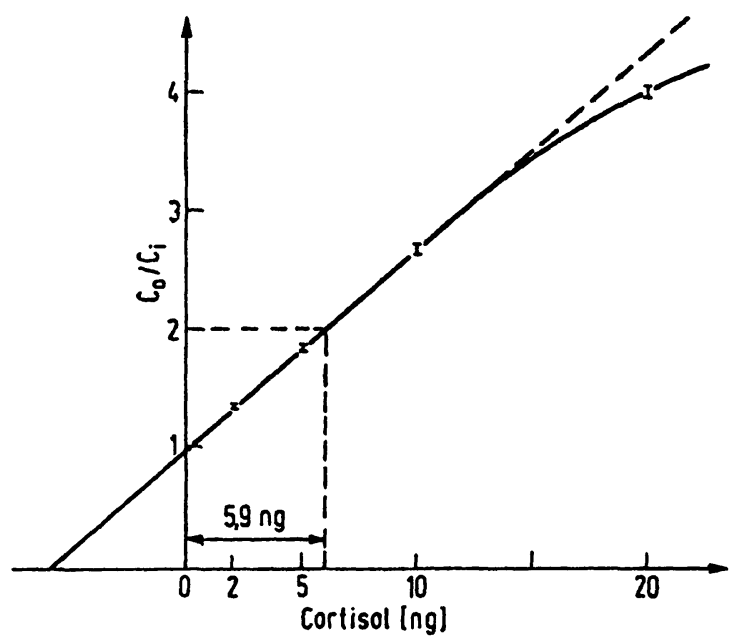

Abb. 3

Dieselbe Standardkurve wie Abbildung 1 in der Darstellungsform $C_{0} / C_{1}$. Der ng-Wert bei dem Quotienten 2 entspricht dem Cortisolgehalt in $1 \mathrm{ml}$ des verdünnten ${ }^{\mathrm{H}} \mathrm{H}$-Cortisol-Bindungsplasmas

Veränderungen der absoluten Aktivitätswerte der Standard-Kurve (etwa durch verlängertes Einwirken der Holzkohle) bleibt diese Gerade weitgehend unverändert. Diese Form der Darstellung bietet die Möglichkeit $z u$ einer automatischen Berechnung der Werte mit Hilfe eines Computers direkt aus den gemessenen Impulsen.

Aus einer Standard-Kurve mit 12fach-Bestimmungen läßt sich für die verschiedenen Bereiche der Kurve die Präzision berechnen (Tab. 1). Sie liegt in allen Bereichen über $95 \%$.

Tab. 1

Präzision der Standardkurve bei $12 \mathrm{fach}$-Bestimmungen in verschiedenen Bereichen

\begin{tabular}{ccc}
\hline $\begin{array}{c}\text { Cortisol } \\
\text { (ng) }\end{array}$ & $\begin{array}{c}\text { s } \\
(\mathrm{ng})\end{array}$ & $\begin{array}{c}\text { VK } \\
(\%)\end{array}$ \\
\hline 0 & $\pm 0,07$ & - \\
2 & $\pm 0,08$ & $4 \%$ \\
5 & $\pm 0,14$ & $2,8 \%$ \\
10 & $\pm 0,22$ & $2,2 \%$ \\
20 & $\pm 0,88$ & $4,4 \%$ \\
\hline
\end{tabular}

Die Sensitivitāt wurde berechnet, indem wir die 2fache Standardabweichung der Nullwerte zugrunde gelegt und den ng-Wert abgelesen haben, der der Impulszahl $C_{0}$, abzüglich des $2 \sigma$-Wertes, entspricht (9). Dies ist der niedrigste Wert, der sich unter Zugrundelegung von $2 \sigma-$ Grenzen von 0 unterscheiden läßt. Danach liegt die Empfindlichkeit der Methode bei 0,14 ng. Wasserextrakte liefern regelmäßig Werte, die von 0 nicht unterscheidbar sind, ein „blank"-Wert braucht also nicht abgezogen zu werden.

Die Reproduzierbarkeit eines 12 fach extrahierten Plasmas mit einem Cortisolgehalt von $130 \mu \mathrm{g} / 1$ innerhalb einer Bestimmungsserie lag bei $\pm 0,6 \mathrm{ng}$, also bei $\pm 4,6 \%$. Bej Abzug der Varianz einer Standardkurve in diesem Bercich errechnet sich für die Plasmaextraktion und die Pipettiervorgänge cin mittlerer Fehlct von $3,7 \%$. Die Streuung von 18 Bestimmungen cincs Mischplasmas von $116 \mu \mathrm{g} / 1$ bei 18 verschiedenen Testansätzen betrug 7,9\%.

Die Extraktion von Cortisol durch Dichlormethan im Verhältnis von 20:1 zu Plasma, gemessen an dem Plasma zugesetzten Tracer-Mengen von ${ }^{3} \mathrm{H}$-Cortisol, unterschied sich nicht signifikant von $100 \%$. Die Pipettierung von $1,0 \mathrm{ml}$ mit der Repetierspritze erfolgt mit ciner Streuung von $1,2 \%$.

Zur Prüfung der Zeitabhängigkeit der Reaktion mit der Holzkohle haben wir eine Reihe von Null-Werten zu verschiedenen Zeiten nach der Zugabe der Holzkohle und dem Schütteln zentrifugiert. In Abbildung 4 ist der Abfall der Impulse/Minute in Abhängigkeit von der

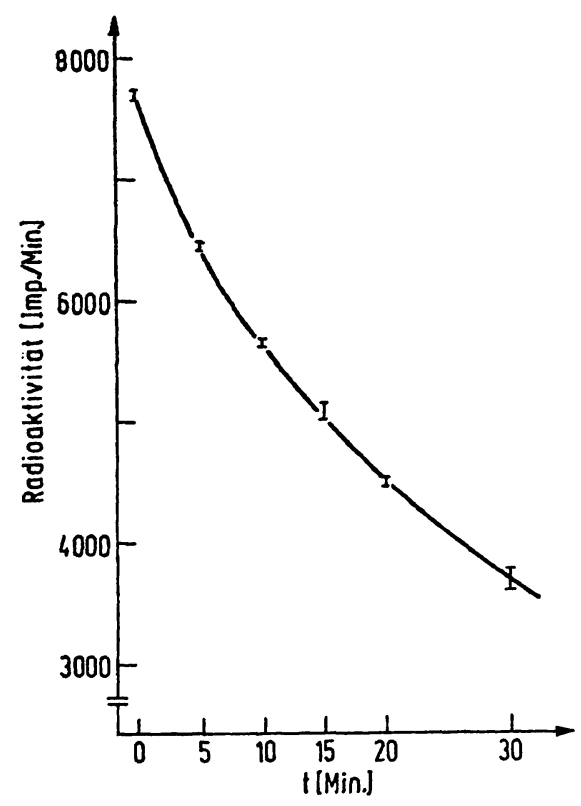

Abb. 4

Zeitabhängigkeit der 'H-Cortisol-Bindung an Protein zwischen $\mathrm{Zu}$ gabe des Dextran-Holzkohlengemisches und Zentrifugieren bei $0^{\circ}$

Zeit wiedergegeben. Im Bereich von $10 \mathrm{Min}$. findet sich ein Abfall von 2,2\%/Min. Da der Pipettiervorgang der Holzkohle-Aufschwemmung eine bestimmte Zeit in Anspruch nimmt, entsteht zwischen dem ersten und dem letzten Reagenzgefäß ein Unterschied in der Reaktionszeit und damit in der Aktivität. Dieser Fehler muß so klein wie möglich gehalten werden. Mit der von uns verwandten Repetierspritze mit automatischer Ansaugung benötigen wir weniger als 40 Sek. zum Pipettieren von 72 Proben. Der Unterschied beträgt dann weniger als 1,5\%, was angesichts der Gesamtvarianz der Methode vertretbar ist. Nach dem Zentrifugieren ist bei im Eisbad stehenden Proben innerhalb von $40 \mathrm{Min}$. keine signifikante Veränderung in der Aktivität zu verzeichnen. Bei Raumtemperatur nimmt die plasmagebundene Aktivität im Laufe von 40 Min. um etwa 10\% ab (Abb. 5). $\mathrm{Da}$ das Abpipettieren des Überstandes etwa $15 \mathrm{Min}$. in Anspruch nimmt, müssen die Proben nach dem Zentrifugieren also erneut in Eiswasser gestellt werden. 


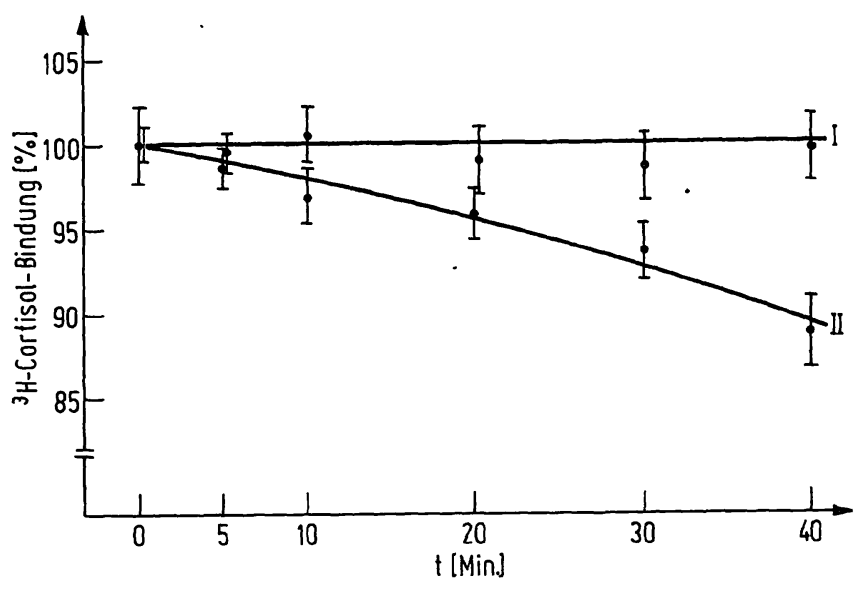

Abb. 5

Zeitabhängigkeit der ${ }^{3} \mathrm{H}$-Cortisol-Bindung an Protein nach dem Zentrifugieren der Holzkohle in \% des Ausgangswertes

II = Raumtemperatur

Im Zusammenhang mit der Testosteronbestimmung nach der Proteinbindungsmethode wurde vorgeschlagen, eine konstante Reaktionszeit mit der Holzkohle dadurch zu erreichen, daß eine dickflüssige Dextranaufschwemmung verwandt wird (10\% Dextran), die das Plasma nach dem Pipettieren zunächst unterschichtet und erst nach dem Schütteln in Reaktion tritt (10). Wir haben diese Methode mit unserer verglichen. Bei $8 \mathrm{fach}-\mathrm{Be}-$ stimmungen je eines 0 -Wertes und eines $10 \mathrm{ng}$-Wertes betrug die Streuung nach unserer Methode 2,06\% bzw. $2,23 \%$, nach der Methode mit visköser Holzkohleaufschwemmung im selben Testansatz $6,80 \%$ bzw. $6,12 \%$.

Die Sensitivität der Methode kann durch stärkere Verdünnung des ${ }^{3} \mathrm{H}$-Cortisol-Bindungsplasmas erhöht werden. Die Grenze der möglichen Plasmaverdünnung wird durch die Abnahme der ${ }^{3} \mathrm{H}$-Aktivität und die nachlassende Zählgenauigkeit gebildet. Diese Aktivität nimmt bei zunehmender Plasmaverdünnung stärker als proportional ab. Wir haben im gleichen Ansatz jeweils 6 „Null-Werte“ mit der üblichen Verdünnung sowie

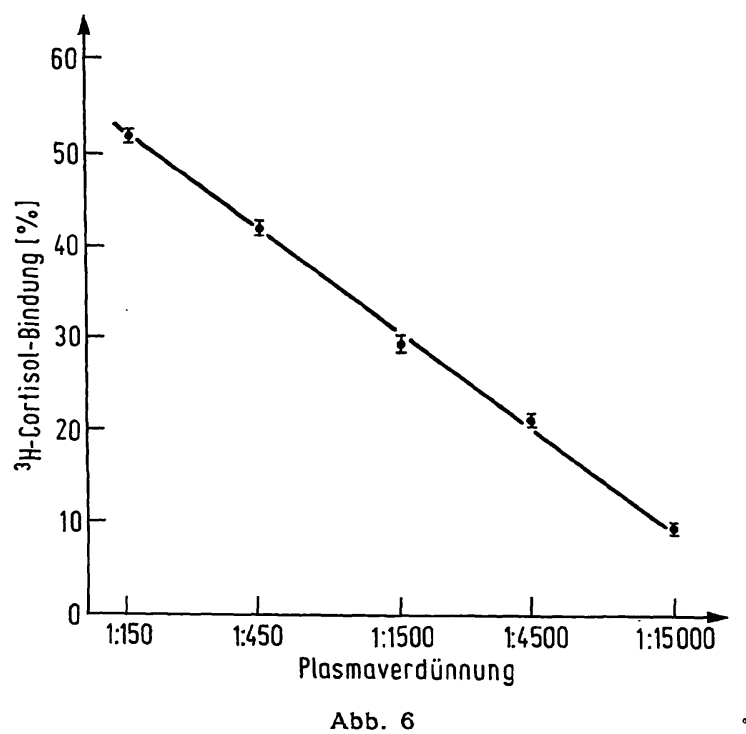

${ }^{3} \mathrm{H}$-Cortisol-Bindung desselben ${ }^{3} \mathrm{H}$-Cortisol-Plasmas ohne Zusatz unmarkierten Cortisols (Null-Wert) bei verschiedenen Verdünnungen weiteren Verdünnungen um den Faktor 1/3, 1/10, 1/30 und 1/100 durchgeführt. Dabei zeigt sich, daß die Bindung an das Plasma nach Inkubation mit der Holzkohle stark abnimmt (Abb. 6). Bei der üblichen Verdünnung von $1 / 150$ sind am Nullpunkt der Kurve etwa 54\% der eingegebenen Aktivität an das Plasma gebunden, bèi einer Verdünnung von 1/15000 nur noch $9 \%$. Die ${ }^{3} \mathrm{H}$-Aktivität am Nullpunkt der Kurve hat also nicht um den Faktor 100, sondern um den Faktor 600 abgenommen.

Wir haben bisher etwa 800 Plasmabestimmungen bei Gesunden und bei Patienten unserer Klinik nach der genannten Methode durchgeführt. Die Normalwerte sind in Tabelle 2 wiedergegeben. Der $2 \sigma$-Bereich wurde

Tab. 2

Normalwerte der Plasma-Cortisol-Bestimmung nach der Proteinbindungsmethode $(\mu \mathrm{g} / 1)$

\begin{tabular}{|c|c|c|c|}
\hline & $\overline{\mathbf{x}}$ & $2 \sigma=$ Bereich & $\mathrm{n}$ \\
\hline \multirow{4}{*}{$\begin{array}{l}\text { Poliklinische Patienten } \\
\text { Stationäre Patienten } \\
\text { (8.00-10.00 Uhr) } \\
\text { Stationäre Patienten } \\
\text { (18.00-22.00 Uhr) } \\
\text { Morgenwerte nach } 2 \mathrm{mg} \\
\text { Dexamethason am Vorabend } \\
2 \text { Stunden nach } 0,25 \mathrm{mg} \\
\text { Synacthen i. v. }\end{array}$} & $\begin{array}{l}115 \\
110\end{array}$ & $\begin{array}{l}70-300 \\
65-233\end{array}$ & $\begin{array}{l}10 \\
99\end{array}$ \\
\hline & 31 & $11-86$ & 44 \\
\hline & 9,5 & $3-31$ & 27 \\
\hline & 345 & $208-573$ & 32 \\
\hline
\end{tabular}

unter der Annahme einer logarithmischen Normalverteilung der Einzelwerte errechnet. Anhand der 99 Normal-Morgenwerte wurde überprüft, daß die Annahme einer logarithmischen Normalverteilung zulässig ist.

Der Streubereich der Werte 12 Stdn. nach $2 \mathrm{mg}$ Dexamethason zeigt keine Überschneidung mit den Ausgangswerten. Die Abendwerte und die Werte nach ACTH zeigen gegenüber den Normal-Morgenwerten eine leichte Überschneidung. Für die Tagesthythmik liegt der mittlere Abfall bei 74,1\% mit einem $2 \sigma$-Bereich von $33,9-89,8 \%(n=44)$. Der Anstieg nach ACTH beträgt im Mittel $225 \%$ mit einem $2 \sigma$-Bereich von $47-621 \%$ $(\mathrm{n}=32)$.

Im Vergleich zur früher bei uns verwandten fluorimetrischen Methode (8) liegen die Normalwerte nach der Proteịnbindungsmethode bei einer beträ̀chtlichen Streuung um durchschnittlich 23\% niedriger. Der Korrelationskoeffizient von 43 Bestimmungen nach beiden Methoden in verschiederien Bereichen beträgt $r=0,792$. Die Regressionsgerade lautet: $y=48,6$ $+0,99 x$ ( $x$ : Protein-Bindungsmethode, $y$ : fluorimetrische Methode). Der relative Anstieg nach ACTH ist bei beiden Methoden etwa gleich. Nach Dexamethason werden bei der fluorimetrischen Methode alle Werte unter $60 \mu \mathrm{g} / 1$ als normal angesehen (obere $2 \sigma$-Grenze). Hier besteht ein deutlicher Unterschied zur ProteinBindungsmethode, bei der alle Werte nach Dexamethason unter $31 \mu \mathrm{g} / \mathrm{l}$ betragen. Auch die Tagesrhythmik war in der Mehrzahl der Fälle bei der fluorimetrischen Methode geringer ausgeprägt, zahlenmäßige Vergleiche wurden jedoch nicht angestellt.

Bei 20 Blutproben mit unterschiedlichem Cortisolgehalt wurde die Extraktion mit Dichlormethan sowohl aus 
Vollblut als auch aus der gleichen Menge von Plasma. durchgeführt. Die Werte aus Vollblut lagen mit breiter Streuung im Mittel um 30,2\% (20-52\%) niedriger. Eine feste Beziehung zum Hämatokrit bestand nicht; die Plasma-Vollblut-Relation variierte auch bei den gleichen Personen bis zu 25\%.

Die Plasmabindung ist nicht spezifisch für Cortisol. Wir prüften die relative Bindungsaffinität gegenüber 11 Desoxy-Cortisol und Progesteron. Eine Standardkurve von 11-Desoxy-Cortisol war von einer gleichzeitig bestimmten Standardkurve von Cortisol nicht zu unterscheiden. Die Progesteron-Standardkurve ist

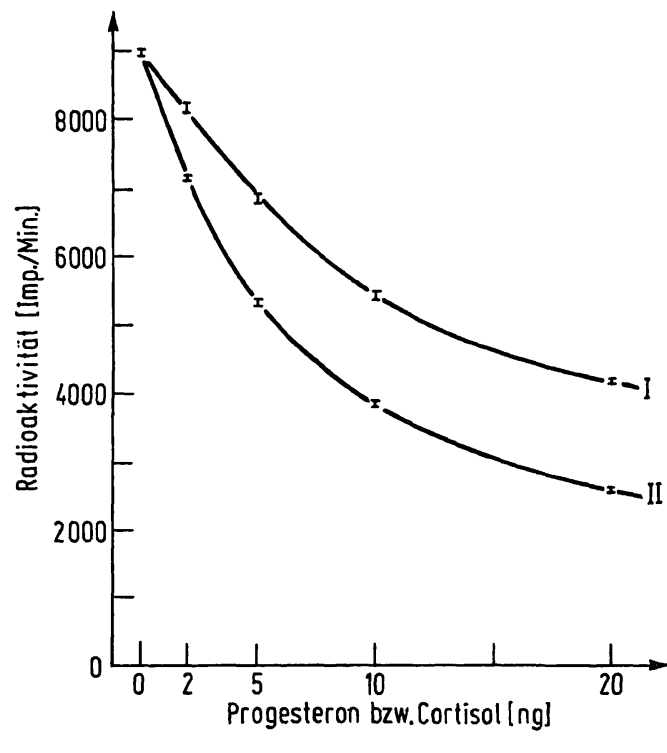

Abb. 7

Standardkurven von Progesteron (I) und Cortisol (II) mit demselben ${ }^{3} \mathrm{H}$-Cortisol-Bindungsplasma (andere Zusammensetzung als in Abb. 1). Die Plasmabindung des Progesteron beträgt etwa $47 \%$ der des Cortisols

deutlich flacher, d. h. die Bindungsaffinität des Plasmas gegenüber Progesteron ist geringer als gegenüber Cortisol. (Abb. 7) Nach den vorliegenden Standardkurven beträgt das Verhältnis 1:0,47. Für die Bestimmung des Plasmaprogesterọns mit unserem System muß also eine Progesteron-Standardkurve benutzt werden.

\section{Diskussion}

\section{Das ${ }^{3} H$-Cortisol-Bindungsplasma}

Für die Steilheit der Verdrängungskurve und damit für die Empfindlichkeit des Systems ist ausschließlich die Gesamtmenge der im Bindungsprotein enthaltenen und im Ansatz konstant zugegebenen Substanz x entscheidend: je geringer sie ist, um so steiler wird die Verdrängungskurve. Der relative Anteil der markierten Substanz (spezifische Aktivität) spielt ebensowenig eine Rolle wie die Menge oder Affinität des Bindungsproteins. Die erste Forderung an ein gutes ${ }^{3} \mathrm{H}$-CortisolBindungsplasma muß also lauten:

1. Die Gesamtmenge der (markierten und unmarkierten)

Substanz $x$ im Ansatz muß möglichst gering sein.

Bei der Darstellung in der Form $C_{0} / C_{1}$ (Abb. 2) bedeutet der Punkt 2, daB die spezifische Aktivität auf die Hälfte abgenommen hat, daß also die zugesetzte Menge der primät eingegebenen entspricht. Die hier abzulesende Menge entspricht also der mit dem Bindungsplasma eingegebenen Gesamtmenge der Substanz $x$. Dieser Wert läßt sich auch (also Negativwert) direkt an der Stelle der $\mathrm{x}$-Achse, wo sie von der Geraden geschnitten wird, ablesen.

Diese einfachen Beziehungen gelten nur, wenn das Bindungsplasma vollständig mit der Substanz $\mathrm{x}$ abgesättigt ist. Anderenfalls wird die Kurve s-förmig und erlaubt im niedrigen Bereich nur eine sehr ungenaue Ablesung (Abb. 1). Die zweite Forderung an das ${ }^{3} \mathrm{H}-$ Cortisol-Bindungsplasma lautet also:

2. Die Gesamtmenge der (markierten und unmarkierten) Substanz x im Ansatz muß größer sein als die Bindungskapazität des Plasmas.

Aufgrund dieser Forderung haben einige Autoren davon abgesehen, vor Entnahme des Bindungsplasmas Dexamethason zur Suppression des eigenen Corticoid-Spiegels zu geben, da dann der Cortisol-Spiegel in der Regel niedriger liegt als die Bindungskapazität. Dabei ist aber übersehen worden, $\mathrm{da} B$ an Stelle des eigenen unmarkierten Cortisols eine entsprechend höhere Menge markierten Cortisols zugegeben werden kann. Dadurch läßt sich eine höhere Aktivität im Ansatz erreichen. Dies ist besonders bei hohen Plasmaverdünnungen für empfindliche Messungen wichtig.

Die dritte Forderung lautet also:

3. Von der Gesamtmenge der Substanz x im Ansatz muß ein möglichst hoher Anteil radioaktiv markiert sein.

Dieses wird am besten erreicht, indem nicht nur der endogene Cortisolspiegel durch Dexamethason gebremst wird, sondern gleichzeitig die Menge an Transcortin durch Östrogen-Medikation (z. B. Ovulationshemmer) erhöht wird.

Die drei genannten Forderungen an ein gutes ${ }^{3} \mathrm{H}-$ Cortisol-Bindungsplasma werden bei der Herstellungsweise wie oben angegeben am besten erfüllt. Die Zugabe von festgelegten Cortisolmengen ohne Austestung des speziellen Plasmas halten wir für weniger günstig.

Der Meßbereich der Methode läßt sich durch stärkere Verdünnung des ${ }^{3} \mathrm{H}$-Cortisol-Bindungsplasmas herabsetzen. Dabei ist die überproportionale Abnahme der ${ }^{3} \mathrm{H}$-Aktivität zu berücksichtigen. (Abb. 6) Worauf diese abnehmende Bindungsaffinität bei Plasmaverdünnungen beruht, ist unklar.

Mit Plasmaverdünnungen bis $1: 2000$ läßt sich noch sehr gut atbeiten, die Sensitivität liegt dann .etwa bei 20 pg. Eine solch hohe Sensitivität ist nur für sehr spezielle Fragestellungen nötig oder wenn andere Steroide (z. B. Progesteron) mit diesem System gemessen werden sollen.

\section{Die Trennung von freiem und gebundenem Cortisol}

Zur Trennung von freiem und gebundenem Anteil von Cortisol werden verschiedenc Methoden angegeben. Gleichgewichtsdialyse (6) und Gelfiltration (11) haben nur noch historisches Interesse. Am verbreitesten ist die Adsorption des freien Cortisols an unlösliche Partikel 
wie Fuller-Erde (4), Florisil $(3,4)$ oder Dextran-Holzkohle $(4,6,7)$. Florisil oder Fuller-Erde werden volumetrisch mit einem Speziallöffel abgemessen. Dieses ist etwas zeitaufwendiger und beinhaltet eine zusätzliche Ungenauigkeit. Die Bindungsfähigkeit von Florisol und Fuller-Erde schwankt von Batch zu Batch erheblich (12). Der Nachteil der Holzkohle liegt darin, daß sie bis zum Zentrifugieren aufgeschwemmt bleibt und in Reaktion tritt. Die Reaktion zwischen der Aktivkohle und dem Liganden (in diesem Fall Cortisol) ist praktisch irreversibel, während die Reaktion zwischen dem Protein und dem Liganden reversibel ist (4). Die Standardisierung der Zeit zwischen Zugabe der Holzkohle und Zentrifugation, also die Konstanthaltung der Kontaktzeit, ist ein kritischer Punkt der Methode (siehe Abb. 3). Daraus ergibt sich, daß der Pipettiervorgang seht schnell gehen muß, damit der Zeitunterschied zwischen der ersten und der letzten Probe möglichst gering ist. Hierzu eignet sich die Repetierspritze Ultra-Asept in hervorragender Weise. Die Notwendigkeit einer Zeitkonstanz begrenzt die Größe der Serien, in der Regel durch die Anzahl der zur Verfügung stehenden Zentrifugenplätze. Die Anwendung von visköser Holzkohleaufschwemmung (10) bringt für die Cortisolbestimmung keinen Vorteil, die Streuungen der Einzelwerte liegen dreimal so hoch wie nach unserer Methode des schnellen Pipettierens von dünnflüssiger Aufschwemmung. Die gleichen Erfahrungen haben wir auch bei der Testosteronbestimmung nach der Proteinbindungsmethode gemacht.

Ein weiterer kritischer Punkt, der für alle Trennmethoden gilt, ist die Konstanthaltung der Temperatur. Die Bindungsaffinität des Transcortin nimmt mit steigender Temperatur stark ab (12-14). Durch das oben angegebene System mit Vorkühlung der Proben, der Holzkohleaufschwemmung, der Repetierspritze und der Zentrifugeneinsätze im Eisbad gelingt es, ohne großen technischen Aufwand die konstante Kühlung bis zum Zentrifugieren aufrechtzuerhalten. Die kurzzeitige Erwärmung während des Zentrifugierens, die alle Proben in gleichem Maße trifft, führt nicht zu einer Beeinträchtigung der Ergebnisse.

\section{Spezifität}

Die Bindung des Cortisols an das sog. Corticosteroidbindende Globulin (CBG, Transcortin) ist nicht spezifisch. Neben Progesteron und 11-Desoxy-Cortisol werden in nennenswertem Umfang Corticosteron, Desoxycorticosteron, 21-Desoxy-Cortisol, 17- $\alpha$-Hydroxyprogesteron und Cortison gebunden $(4,5,7)$. Die relativen Bindungsaffinitäten dieser Steroide gegenüber Cortisol sind stark vom Grade der Plasmaverdünnung abhängig (5). Die Konzentration der genannten Substanzen ist im menschlichen Plasma normalerweise jedoch so gering, daß Störungen selten zu erwarten sind. Sie könnten aber bei Fällen von adrenogenitalen Syndromen mit Vermehrung von Corticosteron, Desoxycorticosteron oder 11-Desoxy-Cortisol auftreten. In der Spätschwangerschaft kann sich auch der hohe Progesteronspiegel bemerkbar machen.
Die fehlende Spezifität schränkt den Wert der Methode kaum ein, erlaubt aber andererseits ihre Anwendung auch für die Bestimmung anderer vom Transcortin gebundener Steroide, z. B. zur Bestimmung des Corticosterons im Rattenplasma. Für die Plasma-ProgesteronBestimmung muß natürlich eine Abtrennung vom Cortisol erfolgen, entweder durch Dünnschicht-Chromatographie $(15,16)$ oder durch eine selektive Extraktion mit bestimmten Chargen von Petroläther (17). Die Bestimmung kann dann mit demselben Bindungsplasma durchgeführt werden, wie es für die Cortisol-Bestimmung verwandt wird. Zur selektiven Bestimmung von 11-Desoxycortisol, z. B. zur Diagnose des 11-Hydroxylasemangels, läßt sich durch eine Benzol-WasserTrennung eine Abgrenzung vom Cortisol durchführen (18). Das Bindungsverhältnis Cortisol zu 11-DesoxyCortisol wurde auch von anderen Autoren bestimmt und mit $1: 0,92$ (5) bzw. 1:0,98 (7) angegeben.

\section{Vergleich mit der fluorimetrischen Methode}

Die nur mäßige UUbereinstimmung bei Doppelbestimmungen von Plasmaproben nach der Proteinbindungsmethode und der fluorimetrischen Methode nach De Moor (8) liegt neben der methodischen Streuung daran, daß mit diesen beiden Methoden etwas Verschiedenes gemessen wird: Die Proteinbindungsmethode erfaßt mehrere Steroide mit unterschiedlicher Bindungsaffinität, ganz überwiegend Cortisol, die fluorimetrische Methode erfaßt 11-Hydroxycorticosteroide, also im wesentlichen Cortisol und Corticosteron sowie eine Reihe unspezifischer Fluorogene, besonders Cholesterin, Cholesterinester und Triglyceride (19). Trotzdem hat die Regressionsgerade zwischen den Doppelbestimmungen die Steigung von 0,99 , was die gute Übereinstimmung für die praktische Anwendung erweist. Der Wert von 48,6 , um den die Regressionsgerade zugunsten der fluorimetrisch gemessenen Werte verschoben ist, ist wahrscheinlich Ausdruck der unspezifischen „Restfluoreszenz". Diese macht sich besonders bei der Bestimmung niedriger Werte (Tagesthythmik, Dexamethasontest) bemerkbar. Hier trennt die Proteinbindungsmethode schärfer vom Normalbereich ab.

Nach unseren Erfahrungen ist die Proteinbindungsmethode der fluorimetrischen an Präzision etwas, an Sensitivität weit überlegen. Auch der Zeitaufwand der Bestimmungen ist geringer. Bei Serien von 72 Proben, davon 5 Standardpunkten in Doppelbestimmungen, verbleiben 62 Proben für 31 Doppelbestimmungen, wobei sich die regelmäßige Kontrolle durch ein Standardplasma empfiehlt. Die 30 Plasmadoppelbestimmungen können von einer technischen Assistentin in 31/2 Stunden durchgeführt werden. Nach 11/2 Stunden Zählzeit wird noch einmal eine halbe bis eine Stunde zum Zeichnen der Standardkurve und zum Errechnen der Werte benötigt.

Ein weiterer Vorteil der Methode, bedingt durch die hohe Sensitivität, ist die Möglichkeit, Bestimmungen aus Kapillarblutentnahmen von $0,1 \mathrm{ml}$ oder weniger durchzuführen. Obgleich nach unseren Untersuchungen Absolutwerte nicht vergleichbar sind, ist die Kapillar- 
blutentnahme mit Bestimmungen aus dem Vollblut zur. Durchführung von Funktionstesten gut geeignet. Dies könnte besonders in der Pädiatrie von Bedeutung sein. Ein weiterer Vorteil der Proteinbindungsmethoden ist ihre Vielseitigkeit. Neben der Anwendbarkeit desselben Bindungsplasmas zur Bestimmung anderer Plasmasteroide ist auch die Bestimmung der freien HarnCorticoide nach Dichlormethan-Extraktion und Reinigung des Extraktes zu nennen (20-22).
Die Proteinbindungsmethoden sind also eine wesentliche Bereicherung für die Analytik von Steroidhormonen. Für die Bestimmung des Plasmacortisols stellen sie unter Beachtung der hier genannten Fehlermöglichkeiten und unter Anwendung der die Durchführung erleichternden Hinweise eine für die Routinediagnostik besonders geeignete Methode von ausreichender Spezifität, hoher Präzision, sehr hoher Sensitivität und relativ geringem Arbeitsaufwand dar.

\section{Literatur}

1. Ekins, R. P., Clin. Chim. Acta, Amsterdam 5, 453 (1960). 2. Diczfalusy, E. (Editor), Steroid assay by protein binding. Transactions of the second symposium on research methods in reproductive endocrinology. Geneva, March 1970. Acta endocr., K'hrn. Suppl. 147 (1970). - 3. Beardwell, C. G., C. W. Burke und C. L. Cope, J. Endocr. 42, 79 (1968). - 4. Murphy, B. E. P., J. Clin. Endocr., Springfield 27, 973 (1967). - 5. Murphy, B. E. P., Recent Progr. Hormone Res. 25, 563 (1969). - 6. Murphy, B. E. P., W. Engelberg und C. J. Pattee, J. Clin. Endocr., Springfield 23, 293 (1963). - 7. Nugent, C. A. und D. M. MaYes, J. Clin. Endocr., Springfield 26, 1116 (1966). 8. De Moor, P., O. Steeno, M. Raskin und A. Hendrikx, Acta Endocr., K'hvn. 33, 297 (1960). - 9. EkINs, R. P. und B. NEwMAN, Acta Endocr., K'hvn. Suppl. 147, 11 (1970). - 10. RoseNFIeld, R. L., W. R. Eberlein und A. M. Bongrovanni, J. Clin. Endocr., Springfield 29, 854 (1969). - 11. Murphy, B. E. P. und C. J. Patree, J. Clin. Endocr., Springfield 24, 919 (1964). -
12. Slaunwhite, W. R. und A. A. Sandberg, Acta Endocr., K'hvn. Suppl. 147, 144 (1970). - 13. Baulieu, E. E., J. P. Raynaud und E. Milgrom, Acta Endocr., K'hvn. Suppl. 147, 104 (1970). - 14. Westrphal, U., Acta Endocr., K'hvn. Suppl. 147, 122 (1970). - 15. Lipsetr, M. B., P. Doerr und J. A. Bermudez, Acta Endocr. K'hvn. Suppl. 147, 155 (1970). 16. Souza, M. L. A., H. O. Williamson, L. O. Moody und E. Diczpalusy, Acta Endocr., K'hvn. Suppl 147, 171 (1970). 17. Jorzansson, E. D. B., Acta Endocr., K'hvn. Suppl. 147, 188 (1970). - 18. Strott, C. A., C. D. West, K. Nakagawa, T. Kondo und H. F. TYLER, J. Clin. Endocr., Springfield 29, 6 (1969). - 19. Graef, V. und H. J. Staudinger, diese Z. 8, 368 (1970). - 20. Hsu, T. H. und T. Bledsoe, J. Clin. Endocr., Springfield 30, 443 (1970). - 21. MeIKLE, A. W., H. TAKIGUCHI, S. Mizutani, F. H. TrleR und C. D. West, J. Laborat. Clin. Med., S. Louis 74, 803 (1969). - 22. Murphy, B. E. P., J. Clin. Endocr., Springfield 28, 343 (1968).
Dr. J. Köbberling 34 Göttingen Humboldtallee 1 